\title{
Orange Peels and Fresnel Integrals
}

\section{Laurent Bartholdi and André Henriques}

C ut the skin of an orange along a thin spiral of constant width (Fig. 1) and place it flat on a table (Fig. 2). A natural breakfast question, for a mathematician, is what shape the spiral peel will have when flattened out. We derive a formula that, for a given cut width, describes the corresponding spiral's shape.

For the analysis, we parametrize the spiral curve by a constant speed trajectory, and express the curvature of the flattened-out spiral as a function of time.

This is achieved by comparing a revolution of the spiral on the orange with a corresponding spiral on a cone tangent to the surface of the orange (Fig. 3, left). Once we know the curvature, we derive a differential equation for our spiral, which we solve analytically (Fig. 4, left).

We then consider what happens to our spirals when we vary the strip width. Two properties are affected: the overall size, and the shape. Taking finer and finer widths of strip, we obtain a sequence of increasingly long spirals; rescale these spirals to make them all of the same size. We show that, after rescaling, the shape of these spirals tends to a well-defined limit. The limit shape is a classical mathematical curve, known as the Euler spiral or the Cornu spiral (Fig. 4, right). This spiral is the solution of the Fresnel integrals.

The Euler spiral has many applications. In optics, it occurs in the study of light diffracting through a slit [1, \$10.3.8]. Let light shine through a long and thin horizontal slit and hit a vertical wall just behind it; assume that the slit's width and the distance to the wall are comparable to the wavelength. What is the illumination intensity on the wall, as a function of height? Imagine two ants running after each other on the Euler spiral at constant speed. It turns out that the square of the distance between the ants, at time $t$, is proportional to the illumination intensity on the wall at height $t$.
The same spiral is also used in civil engineering: it provides optimal curvature for train tracks between a straight run and an upcoming bend [4, \$14.1.2]. A train that travels at constant speed and increases the curvature of its trajectory at a constant rate will naturally follow an arc of the Euler spiral.

The review [2] describes the history of the Euler spiral and its three independent discoveries.

For the purpose of our mathematical treatment, we shall replace the orange by a sphere of radius one. The spiral on the sphere is taken to be of width $1 / N$, as in Fig. 5. The area of the sphere is $4 \pi$, so the spiral has a length of roughly $4 \pi$ $N$. We describe the flattened-out orange-peel spiral by a curve $(x(t), y(t))$ in the plane, parametrized at unit speed from time $t=-2 \pi N$ to $t=2 \pi N$.

On a sphere of radius 1 , the area between two horizontal planes at heights $b_{1}$ and $b_{2}$ is $2 \pi\left(b_{2}-b_{1}\right)$ (see Fig. 5). It follows that, at time $t$, the point on the sphere has height $s:=t / 2 \pi N$.

Our first goal is to find a differential equation for $(x(t), y(t))$. For that, we compute the radius of curvature $R(t)$ of the flattened-out spiral at time $t$ : this is the radius of the circle with best contact to the curve at time $t$. For example, $R(-2 \pi N)=R(2 \pi N)=0$ at the poles, and $R(0)=$ $\infty$ at the equator.

For $N$ large, the spiral at time $t$ follows roughly a parallel at height $s$ on the orange. The surface of the sphere can be approximated by a tangent cone whose development on the plane is a disk sector (Fig. 3, left). The radius

$$
R(t)=\sqrt{1-s^{2}} / s=\sqrt{(2 \pi N)^{2}-t^{2}} / t
$$

of that disk equals the radius of curvature of the spiral at time $t$ (Fig. 3, right). The radius $R(t)$ is in fact only determined up to sign; the construction on one hemisphere

Partially supported by the Courant Research Centre "Higher Order Structures" of the University of Göttingen. 


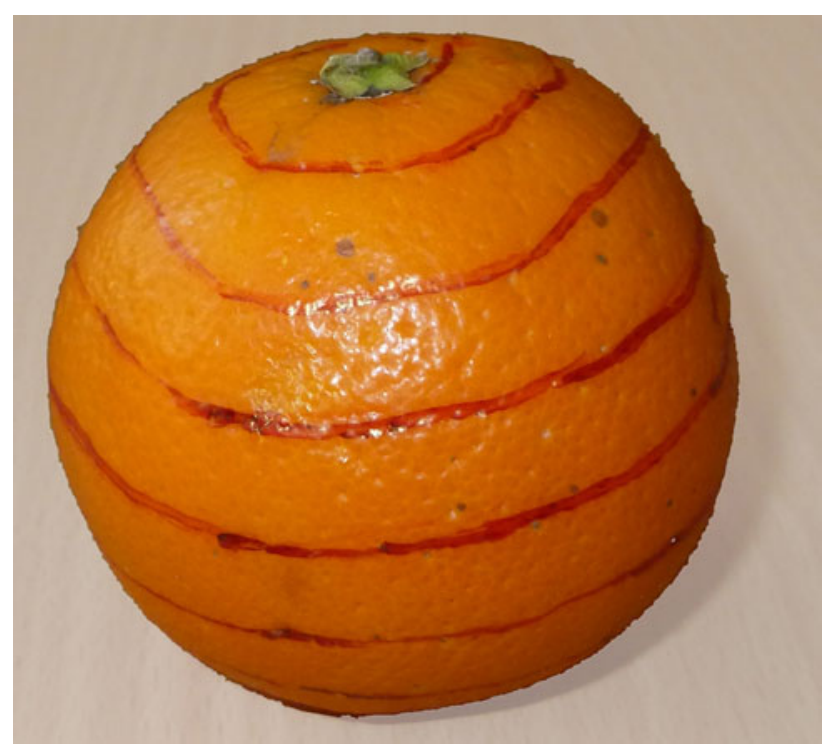

Figure I. An orange, assumed to be a sphere of radius 1, and spiral of width $1 / N$, with $N=3$.

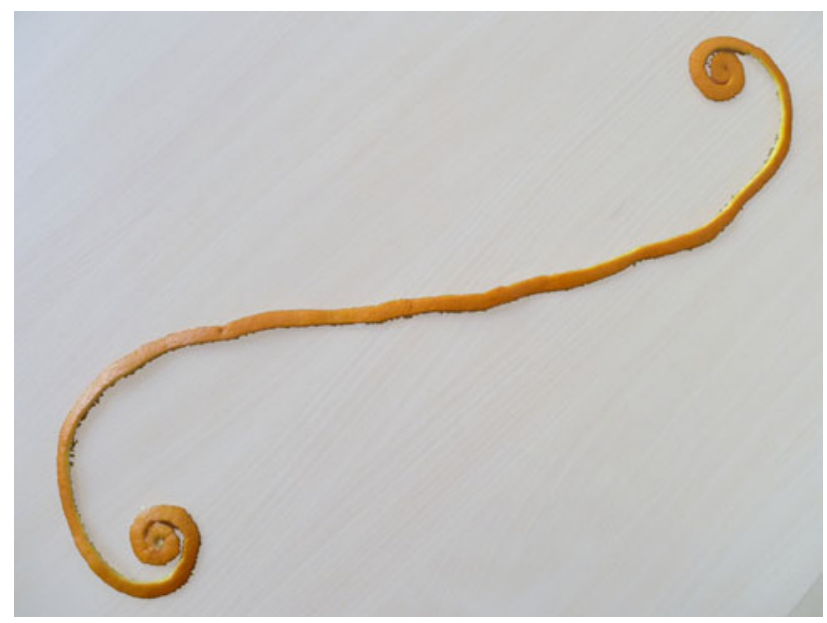

Figure 2. The orange peel in Figure 1 now flattened out.
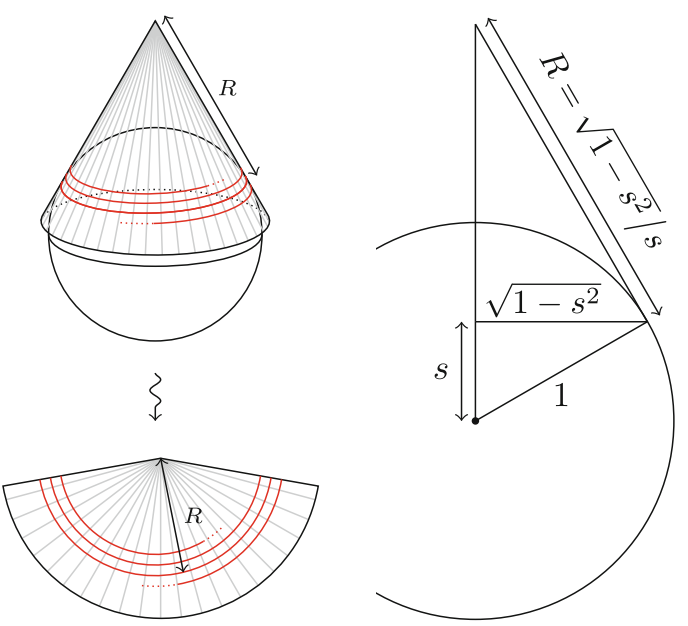

Figure 3. (left) Spiral on the sphere, transferred to the tangent cone and developed on the plane, for computing its radius of curvature. (right) The computation of the radius of curvature $R$ of the flattened spiral.
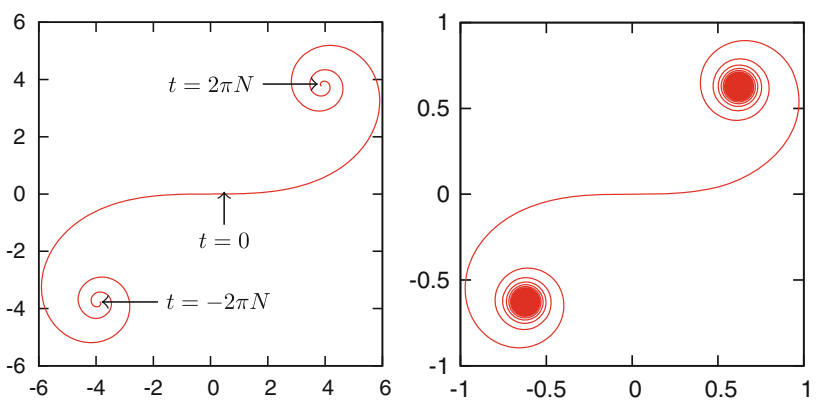

Figure 4. (left) Maple plot of the orange peel spiral $(N=3)$. (right) The Euler spiral: $\lim N \rightarrow \infty$

produces a spiral curling one way, and curling the opposite way on the other.

Now, the condition that we move at unit speed on the sphere - and on the plane - is $(\dot{x})^{2}+(\dot{y})^{2}=1$, and the condition that the spiral has a curvature of $R(t)$ is

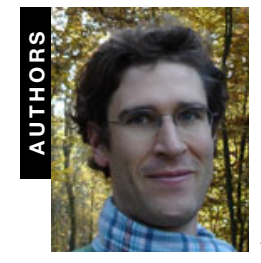

LAURENT BARTHOLDI is Professor of Mathematics at the University of Göttingen. Apart from recreational mathematics, he is interested in group theory, abstract algebra, and complex dynamics. He enjoys playing the piano and harpsichord, and, when in mountainous areas, the alphorn.

Mathematiches Institut

Georg-August Universität zu Göttingen

Göttingen

Germany

e-mail: laurent.bartholdi@gmail.com

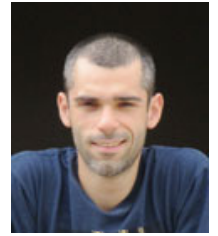

ANDRÉ HENRIQUES is Professor at the University of Utrecht. He is a topologist at heart, even though his research has been scattered across various areas of mathematics, including combinatorics, representation theory, category theory, and operator algebras. He recently spent a year away from his research in order to teach math classes at a local high school.

Mathematisch Instituut

Universiteit Utrecht

Utrecht

The Netherlands

e-mail: a.g.henriques@uu.nl 


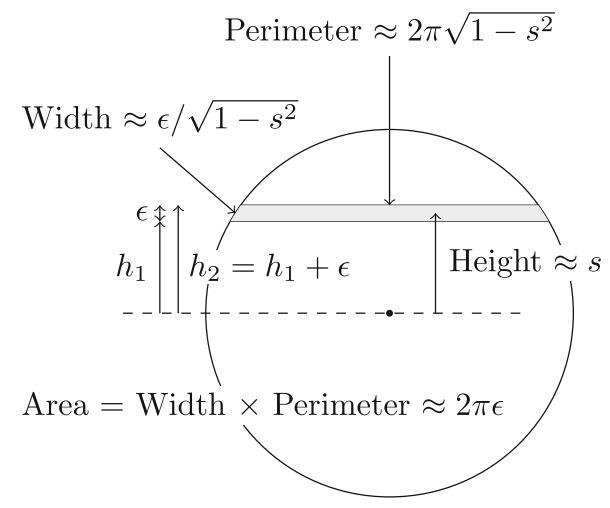

Figure 5. Area of a thin circular strip on the sphere.

$\dot{x} \ddot{y}-\ddot{x} \dot{y}=1 / R$. Here, $\dot{x}$ and $\dot{y}$ are the derivatives of $x$ and $y$, respectively, and $\ddot{x}$ and $\ddot{y}$ are their second derivatives. In fact, introducing the complex path $z(t)=x(t)+i y(t)$, the conditions can be expressed as $|\dot{z}|^{2}=1$ and $\ddot{z} \dot{\bar{z}}=i / R$.

The solution has the general form

$$
z(t)=\int_{0}^{t} \exp (i \phi(u)) d u,
$$

for a real function $\phi$; indeed, its derivative is computed as $\dot{z}=\exp (i \phi(t))$ and has norm 1. As $\ddot{z} \dot{\bar{z}}=i \dot{\phi}(t)$, we have, substituting the expression for $1 / R, \dot{\phi}(t)=s / \sqrt{1-s^{2}}$, which has as elementary solution $\phi(t)=-\sqrt{(2 \pi N)^{2}-t^{2}}$. We have deduced that the flattened-out spiral has parametrization

$$
\left\{\begin{array}{c}
x(t)=\int_{0}^{t} \cos \sqrt{(2 \pi N)^{2}-u^{2}} d u, \\
y(t)=-\int_{0}^{t} \sin \sqrt{(2 \pi N)^{2}-u^{2}} d u .
\end{array}\right.
$$

The flattened-out peel of an orange is shown in Fig. 2, and the corresponding analytic solution, computed by Maple [3], is shown in Fig. 4, left. The orange's radius was $3 \mathrm{~cm}$, and the peel was $1 \mathrm{~cm}$ wide, yielding $N=3$.

What happens if $N$ tends to infinity, that is, if we peel the orange with an ever thinner spiral? For that, we recall the power series approximation

$$
\sqrt{a^{2}-u^{2}}=a-\frac{u^{2}}{2 a}+\mathcal{O}\left(\frac{u^{4}}{a^{3}}\right)
$$

which we substitute with $a=2 \pi N$ in the above expression:

$$
\begin{aligned}
z(t) & =\int_{0}^{t} \exp \left(-i \sqrt{(2 \pi N)^{2}-u^{2}}\right) d u \\
& \approx \int_{0}^{t} \exp \left(-i\left(2 \pi N-\frac{u^{2}}{2 \cdot 2 \pi N}\right)\right) d u .
\end{aligned}
$$

Taking only values of $N$ that are integers, this simplifies to $\int_{0}^{t} \exp \left(i u^{2} / 4 \pi N\right) d u$. We then set $v=u / \sqrt{4 \pi N}$ to obtain

$$
z(t) \approx \sqrt{4 \pi N} \int_{0}^{t / \sqrt{4 \pi N}} \exp \left(i v^{2}\right) d v .
$$

The approximation error is $\int_{0}^{t} \mathcal{O}\left(\frac{u^{4}}{a^{3}}\right) d u=\mathcal{O}\left(t^{5} / N^{3}\right)$, which becomes negligible compared to the size $\mathcal{O}(\sqrt{N})$ of the spiral for $|t| \ll N^{0.7}$.
The above curve is, up to scaling and parametrization speed, the solution of the classical Fresnel integral

$$
(X(t), Y(t))=\left(\int_{0}^{t} \cos u^{2} d u, \int_{0}^{t} \sin u^{2} d u\right)
$$

defined by the condition that the radius of curvature at time $t$ is $1 / 2 t$; here the parametrization is over $t$ from $-\infty$ to $+\infty$. The corresponding curve is called the Euler spiral and winds infinitely often around the points $\pm\left(\sqrt{\frac{\pi}{8}}, \sqrt{\frac{\pi}{8}}\right)$. Setting $T:=t / \sqrt{4 \pi N}$, the condition $|t| \ll N^{0.7}$ becomes $|T| \ll$ $N^{0.2}$. We have thus proven

THeORem If $T \ll N^{0.2}$, then the part of the orange peel of width $1 / N$ parametrized between $-\sqrt{4 \pi N} T$ and $\sqrt{4 \pi N} T$ is a good approximation for the part of the Euler spiral parametrized between $-T$ and $T$ (which corresponds to $T^{2} / 2 \pi$ revolutions on each side of the spiral).

Note that for large $N$, the piece of the orange peel parametrized between $-\sqrt{4 \pi N} T$ and $\sqrt{4 \pi N} T$ forms a rather thin band (of width $\ll N^{-0.3}$ ) around the orange's equator. The aforementioned approximations we made do not apply to the rest of the orange. When the part of the peel contained in that thin band is unrolled, it covers up to $N^{0.4}$ revolutions of the Euler spiral.

As a consequence, the Euler spiral is the limit shape of a wide class of flattened peels: if you take any (convex) fruit that looks like a sphere in a neighborhood of its equator, then the flattened peel of that fruit will tend to the Euler spiral as the peel becomes thinner. More generally, we conjecture that any smooth convex body with positive curvature will share that same property.

The Euler spiral is a well-known mathematical curve. In this article, we explained how to construct it with an orange and a kitchen knife. Flattened fruit peels have already been considered, for example, those of apples [5], but they were never studied analytically. The Euler spiral has had many discoveries across history [2]; ours occurred over breakfast.

\section{OPEN ACCESS}

This article is distributed under the terms of the Creative Commons Attribution License which permits any use, distribution, and reproduction in any medium, provided the original author(s) and the source are credited.

\section{REFERENCES}

[1] Eugene Hecht, Optics, 4th, Pearson Educat., 2002.

[2] Raph Levien, The Euler spiral: a mathematical history, Technical Report UCB/EECS-2008- 111, EECS Department, University of California, Berkeley, 2008.

[3] Michael B. Monagan, Keith O. Geddes, K. Michael Heal, George Labahn, Stefan M. Vorkoetter, James McCarron, and Paul DeMarco, Maple 10 Programming Guide, Maplesoft, Waterloo ON, Canada, 2005.

[4] Vassilios A. Profillidis, Railway management and engineering, Ashgate Publishing Ltd., 2006.

[5] F. M. Turrell, The definite integral symbol, Amer. Math. Monthly 67 (1960), no. 7, 656-658. 